\title{
Erratum
}

\section{Erratum: Nadig et al., Carriage of Supernumerary Sex Chromosomes Decreases the Volume and Alters the Shape of Limbic Structures}

In the article "Carriage of Supernumerary Sex Chromosomes Decreases the Volume and Alters the Shape of Limbic Structures," by Ajay Nadig, Paul K. Reardon, Jakob Seidlitz, Cassidy L. McDermott, Jonathan D. Blumenthal, Liv S. Clasen, Francois Lalonde, Jason P. Lerch, M. Mallar Chakravarty, and Armin Raznahan, which appeared as e0265-18 and published online on October 19, 2018, the authors note that, "It was discovered that deviations from allometric predictions of vertex-wise surface area were incorrectly computed, causing subtle

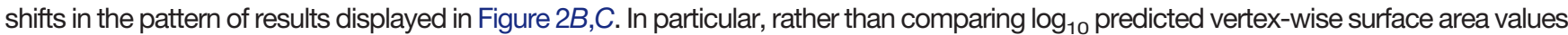
to $\log _{10}$ empirical vertex-wise surface area values, a coding error inadvertently compared $\log _{10}$ predicted vertex-wise surface area values to In empirical vertex-wise surface area values. This change does not affect the main conclusions of the paper, but we have updated Figure 2 and portions of the Abstract, Results, and Discussion describing the spatial distribution of SCA-associated significant vertex-wise surface effects. We sincerely apologize for this mistake."

https://doi.org/10.1523/ENEURO.0039-19.2019

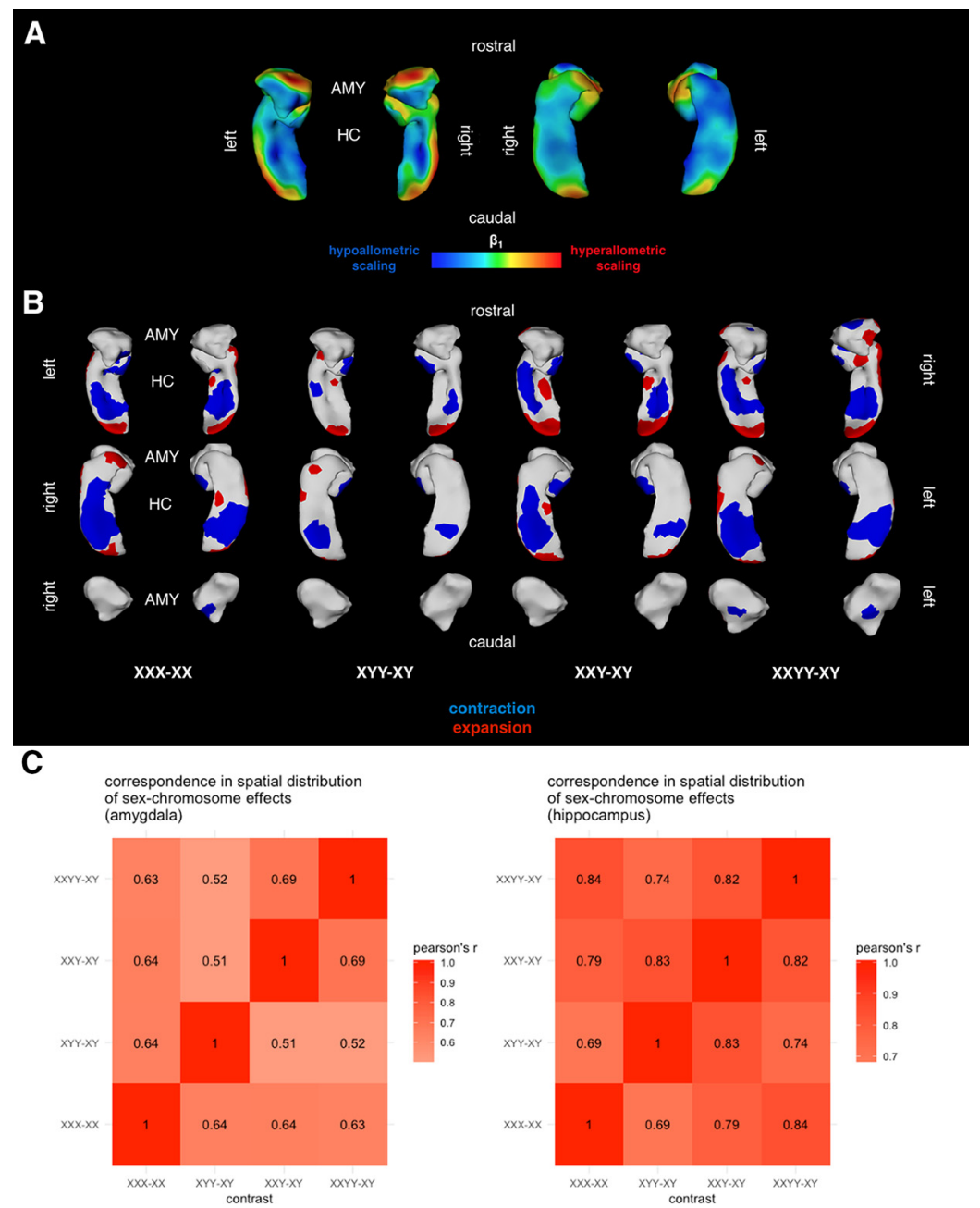

Figure 2. 